\title{
LOW-SENSITIVITY SAB BAND-PASS ACTIVE-RC FILTER USING IMPEDANCE TAPERING
}

\author{
D. Jurišić, G. S. Moschytz, N. Mijat \\ Faculty of Electrical Engineering and Computing \\ Unska 3 \\ Zagreb 10000, CROATIA
}

\begin{abstract}
The design procedure of low-sensitivity second-and third-order class-4 Sallen and Key active resistance-capacitance $(R C)$ allpole filters, using impedance tapering, has already been published. There a ladder structure in the positive feedback loop of an operational amplifier was used. In this paper desensitization using impedance tapering is applied to class-3 circuits with negative feedback referred to as a "single-amplifier biquad" (SAB). The $R C$-section in the negative feedback loop is impedance scaled upwards, from the driving source to the negative amplifier input. Second-order band-pass filters are considered. The improvement comes free of charge; component count and topology remain unchanged, whereas the component values, selected for impedance tapering, account for the significant decrease in sensitivity to component tolerances.
\end{abstract}

\section{INTRODUCTION}

A procedure for the design of class-4 Sallen-and-Key [3] lowsensitivity allpole filters has been presented in [1]. The class-4 filter circuit has an $R C$-ladder network in the positive feedback loop (see [4]). The design presented in [1] is based on "impedance tapering".

In this paper we apply impedance tapering to class-3 allpole active- $R C$ filters. The filter circuit is class-3 referred to as a "single-amplifier biquad" (SAB), and has an $R C$ bridged- $\mathrm{T}$ network in the negative feedback loop as shown in [4]. It is shown here that by the use of impedance tapering, in which Lsections of the $R C$ network are successively impedance scaled upwards, from the driving source to the negative amplifier input, the sensitivity of the filter characteristics to component tolerances can be significantly decreased, in comparison to standard designs. In this paper we consider second-order bandpass filters; the results apply also to other class-3 filter circuits.

It has been shown in [6] that the active building blocks based on positive feedback (class-4) and those based on negative feedback (class-3) are related by the complementary transformation. As a consequence their pole-sensitivity characteristics are closely related, as well as their gain-sensitivity products (GSP) with respect to amplifier gain. This fact can be helpful in applying impedance tapering to $\mathrm{SAB}$ sections.

The improvement in sensitivity achieved by impedance tapering comes free of charge, in that it requires simply the selection of appropriate component values. The design procedure adds nothing to the cost of conventional circuits; component count and topology remain unchanged, whereas the component values selected for impedance tapering considerably decrease component tolerance sensitivity. This is demonstrated by Monte Carlo analysis (using PSPICE simulation) to examine the sensitivity of a band-pass filters transfer function to component tolerances. A simple Voltage-Controlled-Voltage-Source (VCVS) was used to model an ideal operational amplifier.

\section{DEFINITION OF SENSITIVITY}

The relative sensitivity of a function $F(x)$ to variations of a variable $x$ is defined as

$$
S_{x}^{F(x)}=\frac{d F / F}{d x / x}=\frac{d F(x)}{d x} \frac{x}{F(x)}=\frac{d[\ln F(x)]}{d[\ln x]} .
$$

Consider the transfer function $T(s)$ of a second-order, allpole band-pass filter expressed in terms of coefficients $a_{i}$

$$
T(s)=\frac{N(s)}{D(s)}=\frac{K a_{1} s}{s^{2}+a_{1} s+a_{0}}
$$

The filter coefficients $a_{i}$ of the polynomial $D(s)$ for given filter specifications are available from any filter handbook. The relative change of $T(s)$ to the variation of its coefficients $a_{i}$ is

$$
\frac{\Delta T(s)}{T(s)}=\sum_{i=0}^{n} S_{a_{i}}^{T(s)} \frac{\Delta a_{i}}{a_{i}}
$$

where $S_{a_{i}}^{T(s)}$ is the sensitivity to coefficient variations, and is dependent only on the value of the coefficients $a_{i}$ and frequency $\omega$. The coefficient variation is given by

$$
\frac{\Delta a_{i}}{a_{i}}=\sum_{\mu=1}^{r} S_{R_{\mu}}^{a_{i}} \frac{\Delta R_{\mu}}{R_{\mu}}+\sum_{\mathrm{v}=1}^{c} S_{C_{\mathrm{v}}}^{a_{i}} \frac{\Delta C_{\mathrm{v}}}{C_{\mathrm{v}}}+S_{\bar{\beta}}^{a_{i}} \frac{\Delta \bar{\beta}}{\bar{\beta}}
$$

where $R_{\mu}$ are resistors, $C_{v}$ capacitors and $\bar{\beta}$ the feedback coefficient of an operational amplifier $(x)$. On the other hand the coefficient-to-component sensitivities $S_{x}^{a_{i}}$, where $x$ represents each of the component types, are dependent on the realisation of the filter circuit and can be reduced by non-standard filter design as shown in [1].

\section{DESIGN OF SECOND-ORDER CLASS-3 BAND-PASS FILTER}

As a representative example we consider a second-order bandpass (R) filter shown in Fig. 1 [5]. It has a bridged-T circuit in the negative feedback loop and is known as a class- 3 band-pass filter. 


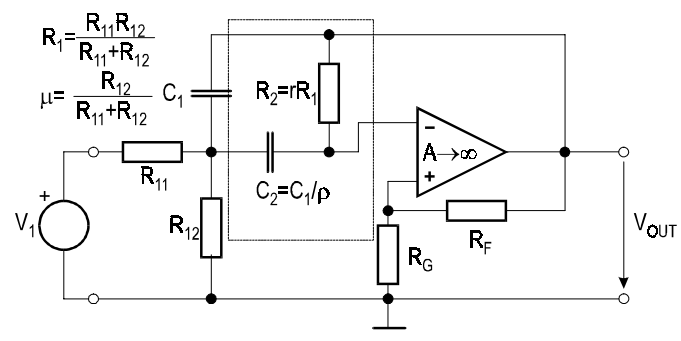

Figure 1. Second-order class-3 band-pass filter (medium-Q realization).

The voltage transfer function $T(s)$ for this circuit, expressed in terms of coefficients $a_{i}$, is given by eq. (2), and in terms of the pole frequency $\omega_{p}$ and pole $\mathrm{Q}, q_{p}$ by

$$
\begin{gathered}
T(s)=\frac{V_{\text {OUT }}}{V_{1}}=\frac{K^{\prime} \omega_{p} s}{s^{2}+\frac{\omega_{p}}{q_{p}} s+\omega_{p}^{2}}, \text { where } K^{\prime}=\mu \cdot \bar{\beta} \sqrt{\frac{R_{2} C_{2}}{R_{1} C_{1}}}, \\
a_{0}=\omega_{p}^{2}=\frac{1}{R_{1} R_{2} C_{1} C_{2}}, a_{1}=\frac{\omega_{p}}{q_{p}}=\frac{R_{1}\left(C_{1}+C_{2}\right)+R_{2} C_{2}-\bar{\beta} R_{2} C_{2}}{R_{1} R_{2} C_{1} C_{2}}, \\
q_{p}=\frac{\sqrt{R_{1} R_{2} C_{1} C_{2}}}{R_{1}\left(C_{1}+C_{2}\right)+R_{2} C_{2}-\bar{\beta} R_{2} C_{2}} \text { and } \bar{\beta}=1+\frac{R_{G}}{R_{F}}=\frac{\beta}{\beta-1} . \text { (5) }
\end{gathered}
$$

where gain $\beta=1+R_{F} / R_{G}$ represents the gain in the complementary class-4 filter circuit, i.e. filter with positive feedback loop as in [1].

The sensitivity of $a_{0}$ to all $R C$ components is -1 (and to the gain $\bar{\beta}$ is zero), thus $\Delta a_{0} / a_{0}$ can be decreased only by decreasing the tolerance of $R_{1}, R_{2}, C_{1}$ and $C_{2}$, i.e. by applying technological measures. This is also true for the filters of class- 4 as shown in [1]. For the sensitivity of $a_{1}$ to the tolerance of the passive components, we readily obtain expressions given in the first column of Table 1.

As already stressed above class-4 (positive feedback) and class-3 (negative feedback) biquads have been called complementary. For example, a class-4 high-pass biquad and a band-pass (class3) SAB filter section, as presented in Fig. 1, have been shown to possess identical poles and identical root loci (with respect to $\beta$ and $\bar{\beta}$, respectively). Both circuits are described in [6]. The important consequence is that the sensitivity reduction to component tolerances of one circuit directly reduces the sensitivity of the other. Thus the pole-Q factor, $q_{p}$ of the highpass class- 4 filter section (with the gain $\beta$ ), is identical to the pole- $Q$ factor of the band-pass $\mathrm{SAB}$ filter section given by eq. (5) (gain $\bar{\beta}$ ). Although the way of minimizing the sensitivity of the coefficient $a_{1}$ with respect to $\beta$, i.e. $S_{\beta}^{a_{1}}$ for class- 4 circuits is known, it will be briefly recapitulated here, and expanded on for the $\mathrm{SAB}$ section. Thus, for both circuits we obtain

$$
S_{\bar{\beta}}^{a_{1}}=-S_{\bar{\beta}}^{q_{p}}=-\left(\frac{q_{p}}{\hat{q}}-1\right)
$$

where we denote the pole $\mathrm{Q}$ of the passive subnetwork by $\hat{q}$. ( $S_{\beta}^{a_{1}}$ is with respect to $\beta$ for class- 4 circuits in $[1], S_{\bar{\beta}}^{a_{1}}$ to $\bar{\beta}$ for the $\mathrm{SAB}$ sections in this paper).

The coefficient sensitivities are all proportional to the pole $\mathrm{Q}, q_{p}$. Thus, as already shown in [1], one does well to select the filter type with the lowest pole Qs for a given application. From (6) it follows that the coefficient $a_{1}$ sensitivity to the gain is inversely proportional to $\hat{q}$, i.e. to the pole $\mathrm{Q}$ factor of the passive network which is limited to less than 0.5 [7]. The value of $\hat{q}$ can be maximized by appropriately impedance-wise scaling individual sections of an $R C$ network from one another. This is referred to as "impedance scaling" in [1]. Referring to Fig. 1, for our circuit this is accomplished when the second $R C$-section in the feedback loop comprising $R_{2}$ and $C_{2}$ (inside the rectangle) is impedance scaled upwards in order to minimize the loading on the first, i.e. $R_{1}$ and $C_{1}$. Letting

$$
R_{1}=R ; \quad C_{1}=C ; \quad R_{2}=r R ; \quad C_{2}=C / \rho
$$

we obtain the sensitivity relations given in the second column of Table 1, and we obtain from (5)

$$
\hat{q}=\left.\frac{\sqrt{r \rho}}{1+r+\rho}\right|_{r=\rho}=\left.\frac{\rho}{1+2 \rho}\right|_{\rho \rightarrow \infty}=0.5
$$

Thus impedance scaling $R_{2}$ and $C_{2}$ as in (7), $\hat{q}$ will approach 0.5 and the sensitivity of $a_{1}$ (or $q_{p}$ ) to $\bar{\beta}$ will be minimized according to (6). A glance at the sensitivities in the second column of Table 1, shows that some of them are proportional to $\rho$ and some to $\rho^{-1}$. Thus, setting $\rho=1$ provides an optimum compromise. Note that $S_{\bar{\beta}}^{a_{1}}=-S_{\bar{\beta}}^{q_{p}}$.

Table 1. Sensitivity of $a_{1}$ to component variations of a second-order class-3 band-pass filter.

\begin{tabular}{c|c|c}
\hline$x$ & \multicolumn{2}{|c}{$-\frac{1}{q_{p}} S_{x}^{a_{1}}$} \\
\hline$R_{1}$ & $-\sqrt{\frac{R_{2} C_{2}}{R_{1} C_{1}}} \cdot(\bar{\beta}-1)$ & $-\sqrt{\frac{r}{\rho}} \cdot(\bar{\beta}-1)$ \\
\hline$R_{2}$ & $\sqrt{\frac{R_{1} C_{1}}{R_{2} C_{2}}}+\sqrt{\frac{R_{1} C_{2}}{R_{2} C_{1}}}$ & $\sqrt{\frac{\rho}{r}}+\frac{1}{\sqrt{r \rho}}$ \\
\hline$C_{1}$ & $\sqrt{\frac{R_{1} C_{2}}{R_{2} C_{1}}}-\sqrt{\frac{R_{2} C_{2}}{R_{1} C_{1}}} \cdot(\bar{\beta}-1)$ & $\frac{1}{\sqrt{r \rho}}-\sqrt{\frac{r}{\rho}} \cdot(\bar{\beta}-1)$ \\
\hline$C_{2}$ & $\sqrt{\frac{R_{1} C_{1}}{R_{2} C_{2}}}$ & $\sqrt{\frac{\rho}{r}}$ \\
\hline
\end{tabular}

Design equations for the tapered second-order band-pass filter follow. With the tapering factors in (7) and with

$$
\omega_{0}=(R C)^{-1}
$$

we obtain for the coefficients of $T(s)$

$$
a_{0}=\frac{\rho \omega_{0}^{2}}{r}, a_{1}=\omega_{0}\left[\frac{\rho+1}{r}-\bar{\beta}+1\right], \bar{\beta}=\frac{\rho+1}{r}-\frac{1}{q_{p}} \sqrt{\frac{\rho}{r}}+1 \text {. }
$$



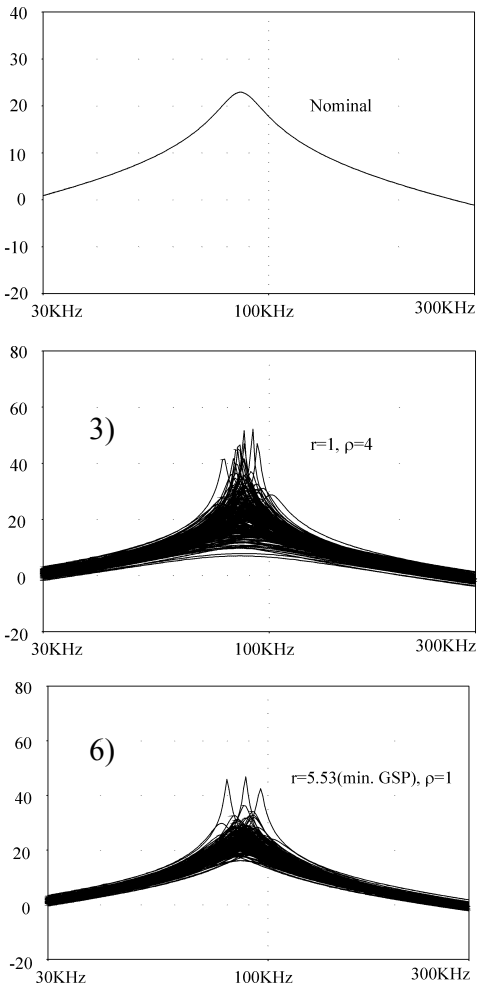
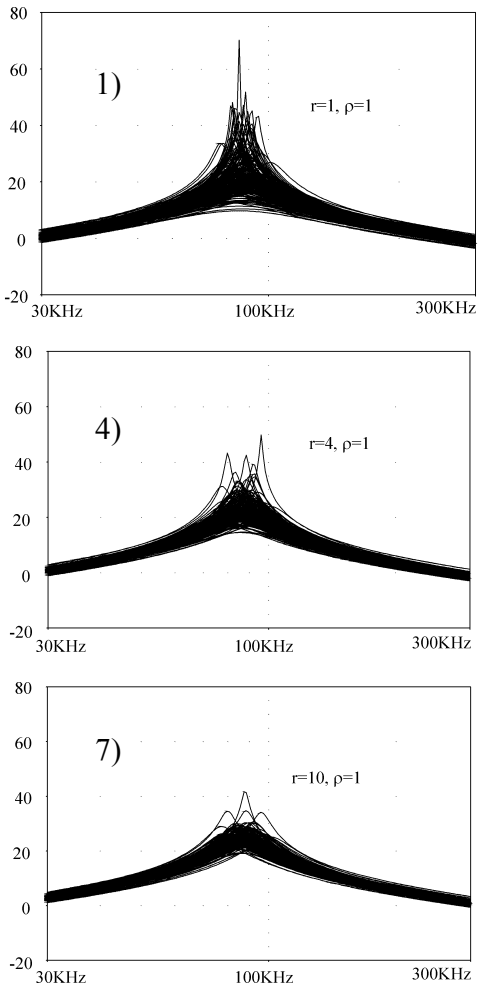
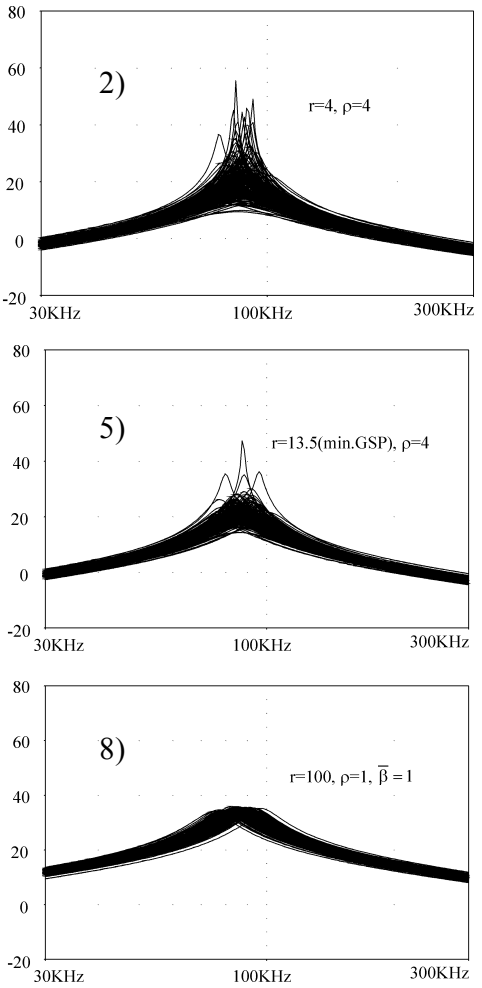

Figure 2. Monte Carlo response plots of impedance-tapered second-order band-pass filters given in Table 2

From $K, a_{0}=\omega_{p}^{2}$ and $a_{1}=\omega_{p} / q_{p}$, which are given by the filter specifications, we must determine $\omega_{0}, \rho, r$ and $\bar{\beta}$. Parameters $r$ and $\rho$ must both be positive, and $\bar{\beta}$ must be larger than unity. Therefore, the constraint that

$$
\omega_{0}>-\frac{a_{1}}{2(\bar{\beta}-1)}+\sqrt{\left(\frac{a_{1}}{2(\bar{\beta}-1)}\right)^{2}+\frac{a_{0}}{(\bar{\beta}-1)}}
$$

Because $\bar{\beta} \geq 1$ the expression under the square root will always be positive.

Note that the gain factor $K$ in eq. (2), where $K=q_{p} \cdot K^{\prime}$, is the filter's gain in the pass band and can be specified by the filter designer, but the amplifier gain $\bar{\beta}$ is determined by the expression in eq. (10).

Thus, from eq. (5), we obtain $\mu$. If the desired value of $\mu$ is less than unity, then the specified gain $K$ can be tuned with a resistive voltage divider at the input of the network, consisting of $R_{11}$ and $R_{12}$, as shown in Fig. 1 . For the value of $\mu>1$ it is possible to make an output voltage-level transformation, this has been described elsewhere (see [1], [5]).

\subsection{Example}

Consider the following practical example. Suppose that

$$
\omega_{p}=2 \pi \cdot 86 \mathrm{kHz} ; q_{p}=5 ; \quad C=500 \mathrm{pF} .
$$

In the design process, various ways of impedance tapering have been applied, the resulting component values are presented in Table 2. Monte Carlo runs with 5\% Gaussian distribution, zeromean resistors and capacitors were carried out for the resulting filters and presented in Fig. 2.

Table 2. Component values of second-order class-3 band-pass filters with various tapering techniques (resistors in $[\mathrm{k} \Omega]$, capacitors in $[\mathrm{pF}]$ ).

\begin{tabular}{c|c|c|c|c|c|c|c}
\hline Nr. & Filter & $R_{1}$ & $r$ & $C_{1}$ & $\rho$ & $\bar{\beta}$ & GSP \\
\hline 1$)$ & Non Tapered & 3.7 & 1 & 500 & 1 & 2.8 & 39.2 \\
\hline 2$)$ & Impedance Tapered & 3.7 & 4 & 500 & 4 & 2.05 & 21.0 \\
\hline 3$)$ & Part. Tapered $(r=1)$ & 7.4 & 1 & 500 & 4 & 5.6 & 78.4 \\
\hline 4$)$ & Part. Tapered $(\rho=1)$ & 1.85 & 4 & 500 & 1 & 1.4 & 19.6 \\
\hline 5$)$ & C-Taper, min. GSP & 2.012 & 13.52 & 500 & 4 & 1.26 & 14.62 \\
\hline 6$)$ & $\rho=1$ and min. GSP & 1.57 & 5.53 & 500 & 1 & 1.28 & 19.2 \\
\hline 7$)$ & Part Tapered $(\rho=1)$ & 1.17 & 10 & 500 & 1 & 1.137 & 20.43 \\
\hline 8$)$ & Part Tapered $(\rho=1)$ & 0.370 & 100 & 500 & 1 & 1.0 & 50.00 \\
\hline
\end{tabular}

Observing the Monte Carlo runs in Fig. 2 one can conclude that the ideally impedance-tapered filter (Nr. 2) and the partiallytapered filter with equal resistors $(r=1)$ (Nr. 3) do not significantly decrease sensitivity with respect to component variations, compared with the non-tapered standard circuit $(\mathrm{Nr}$. 1). The C-tapered and min. GSP filter (Nr. 5) shows somewhat lower sensitivity. Furthermore the circuit Nr. 6) with $r$ for min. GSP has a higher sensitivity than circuits Nr. 7), 8) for which $r$ is larger but the GSP is not minimized. 
The minimum sensitivity of the SAB section is achieved by selecting equal capacitors $(\rho=1)$ and tapering the resistors. This corresponds to the minimum sensitivity achieved with resistive tapering and $\rho=1$ of the high-pass class- 4 circuit. This is because the high-pass class- 4 circuit and the band-pass SAB circuit are complementary [6]. A special case is the circuit Nr. 8) with the very large value of $r=100$ and the gain $\bar{\beta}=1$ (note that for $\rho=1$ with increasing of $r: \bar{\beta} \rightarrow 1$ ). It has the minimum sensitivity. This realization is suitable for low-Q pole factors, $q_{p}$ and is presented in Fig. 3 and in [5].

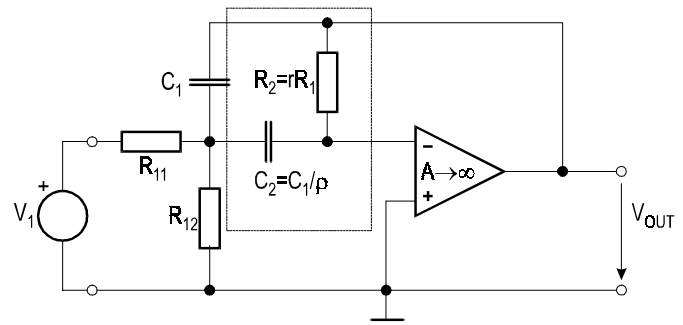

Figure 3. Second-order class-3 band-pass filter with unity gain $\bar{\beta}$ (low-Q realization).

In summary, for the general second-order allpole Class-3 bandpass filter, resistive impedance tapering with equal capacitors $(\rho=1)$, provides low sensitivity circuits. With lower values of $Q-$ pole factors, $q_{p}$, the circuit with unity gain $\bar{\beta}$ has minimum sensitivity to the component tolerances of the circuit.

\subsection{Impedance Tapering of Resistors with $\rho=1$}

This can be accomplished by the following step-by-step design procedure, for given filter specifications, i.e. $K, \omega_{p}$ and $q_{p}$ :

i) With $\rho=1$, select $r$ and calculate $\bar{\beta}$ : Let $r=10$, thus

$$
\bar{\beta}=2 / r-1 /\left(q_{p} \sqrt{r}\right)+1=2 / 10-1 /(5 \sqrt{10})+1=1.137 .
$$

ii) Select $R_{G}$ and calculate $R_{F}$ : Let $R_{G}=10 \mathrm{k} \Omega$, then

$$
R_{F}=R_{G} /(\bar{\beta}-1)=10 \mathrm{k} \Omega / 0.137=73 \mathrm{k} \Omega .
$$

iii) Calculate $\omega_{0}: \omega_{0}=\sqrt{r} \omega_{p}=\sqrt{10} 2 \pi 86 \mathrm{kHz}=1.709 \cdot 10^{6} \mathrm{rad} / \mathrm{s}$.

iv) Select $C_{1}$ and compute $R_{11}, R_{12}$ and $R_{2}$ :

Let $C=500 \mathrm{pF}$ thus $C_{1}=C_{2}=C=500 \mathrm{pF}, R=\left(\omega_{0} C\right)^{-1}=\left(1.708 \cdot 10^{6}\right.$. $\left.500 \cdot 10^{-12}\right)^{-1}=1.17 \mathrm{k} \Omega$. For a given pass-band gain $K=5$, the value $\mu=K /\left(q_{p} \bar{\beta} \sqrt{r}\right)=5 /(5 \cdot 1.137 \cdot \sqrt{10})=0.287<1$. Instead of $R_{1}=R=$ $=5.853 \mathrm{k} \Omega$ there is a voltage attenuator at the signal input consisting of $R_{11}=R_{1} / \mu=1.17 \mathrm{k} \Omega / 0.278=4.2 \mathrm{k} \Omega$ and $R_{12}=R_{1} /(1-\mu)=$ $=1.17 \mathrm{k} \Omega / 0.722=1.62 \mathrm{k} \Omega$. Then $R_{2}=r R=11.7 \mathrm{k} \Omega$. (Filter Nr. 7)

v) Compute the GSP: GSP $=q_{p} \bar{\beta}^{2} \sqrt{r}=5 \cdot 1.137^{2} \cdot \sqrt{10}=20.43$.

\subsection{Resistive Tapering with $\rho=1$ and $\bar{\beta}=1$}

As discussed above, the design with $\bar{\beta}=1$ refers to the circuit in Fig. 3. The design can be carried out by the following step-bystep design procedure:

i) For a given $\rho=1$ calculate $r: r=4 \cdot q_{p}^{2}=4 \cdot 5^{2}=100$.

ii) Calculate $\omega_{0}: \omega_{0}=\sqrt{r} \omega_{p}=\sqrt{100} 2 \pi 86 \mathrm{kHz}=5.4 \cdot 10^{6} \mathrm{rad} / \mathrm{s}$. iii) Select $C_{1}$ and compute $R_{11}, R_{12}$ and $R_{2}$ : Let $C=500 \mathrm{pF}$ thus $C_{1}=C_{2}=C=500 \mathrm{pF}, R=\left(\omega_{0} C\right)^{-1}=\left(5.4 \cdot 10^{6} \cdot 500 \cdot 10^{-12}\right)^{-1}=370.13 \Omega$.

For $K=5, \mu=K /\left(q_{p} \bar{\beta} \sqrt{r}\right)=5 /(5 \cdot 1 . \quad \sqrt{10})=0.1<1 ; \quad R_{1}=R=$ $370.13 \Omega, R_{11}=R_{1} / \mu=370.13 \Omega / 0.1=3.7 \mathrm{k} \Omega$ and $R_{12}=R_{1} /(1-\mu)=$ $370.13 \Omega / 0.9=411.25 \Omega ; R_{2}=r R=37.01 \mathrm{k} \Omega$. (Filter circuit Nr. 8) iv) Compute the GSP: GSP $=q_{p}^{2}(1+1 / \rho)=25 \cdot 2=50$.

This circuit has the minimum sensitivity to component tolerances. To choose between a "medium-Q" circuit $(\bar{\beta}>1)$ as in Fig. 1, and a "low-Q" circuit $(\bar{\beta}=1)$ as in Fig. 3, there is only one criterion, i.e. the value of Q-pole factor, $q_{p}$. The value of $r$ is proportional to the squared value of $q_{p}$ (see [5]). The problem arises for higher values of $q_{p}$, say $q_{p}>5$. For the value of Q-pole factor, $q_{p}=5$, circuits with $\bar{\beta}=1$ have resistor tapering factors $r$, which are technologically hardly feasible. For example $\rho=1$, with $q_{p}=5$ yields the value of $r=100$.

\section{SUMMARY}

A procedure for the design of low-sensitivity active resistancecapacitance $(R C)$ allpole filters of second- and third-order has already been published [1]. In this paper a procedure for the design of band-pass SAB circuits as given, for example, in [5] is presented. Instead of a standard design method, the component values are calculated using "impedance tapering". It has been shown that class-4 circuits and the class-3 SAB sections are complementary [6]. Thus desensitization by impedance tapering of one section produces the same desensitization of the other. Resistive impedance tapering with equal capacitors $(\rho=1)$ provides circuits with minimum sensitivity to the component tolerances. In particular, the circuits with unity gain have minimum sensitivity to the component tolerances of the circuit.

\section{REFERENCES}

[1] G. S. Moschytz, "Low-Sensitivity, Low-Power, Active-RC Allpole Filters Using Impedance Tapering," IEEE Trans. on Circuits and Systems, vol. CAS-46, no. 8, pp. 1009-1026, Aug. 1999.

[2] G. S. Moschytz, "Realizability constraints for third-order impedance-tapered allpole filters," IEEE Trans. on Circuits and Systems, vol. CAS-46, no. 8, pp. 1073-1077, Aug. 1999.

[3] R. P. Sallen and E. L. Key, "A practical Method of Designing $R C$ Active Filters," IRE Transactions on Circuit Theory, vol. CT-2, pp. 78-85, 1955.

[4] G. S. Moschytz, Linear Integrated Networks: Design. New York (Bell Laboratories Series): Van Nostrad Reinhold Co., 1975.

[5] G. S. Moschytz and P. Horn, Active Filter Design Handbook. Chichester, U.K.: Wiley 1981.

[6] G. S. Moschytz and P. Horn, "Optimizing two commonly used active-filter building blocks using the complementary transformation," Electronic Circuits and Systems, vol. 1, no. 4, pp. 125-132, July 1977.

[7] G. S. Moschytz, Linear Integrated Networks: Fundamentals. New York (Bell Laboratories Series): Van Nostrad Reinhold Co., 1974. 